\title{
Molecular Characterization of an H5N3 Influenza Virus Isolated from Spot-Billed Duck
}

\author{
Jin Hwa Lee ${ }^{1}$, Hyuk Moo Kwon ${ }^{1}$ and Haan Woo Sung ${ }^{1^{+}}$ \\ ${ }^{I}$ College of Veterinary Medicine, Kangwon National University, Chuncheon, 200-701, Republic of Korea.
}

\begin{abstract}
Among the 16 hemagglutinin (HA) subtypes of avian influenza virus (AIV), only the H5 and H7 subtypes have caused highly pathogenic avian influenza (HPAI) in poultry. However, most $\mathrm{H} 5$ or H7 subtype viruses are categorized as low pathogenic avian influenza (LPAI). Some AIVs, including the H5 and H7 HPAI viruses, have shown the ability to infect humans directly. In this study, we describe the biological and molecular characterization of an H5N3 AIV (SBD/KR/KNU SYG06/06) isolated from spot-billed duck (Anas poecilorhyncha) in Korea. A phylogenetic analysis of the eight viral genes showed that the SBD/KR/KNU SYG06/06 isolate belongs to the Eurasian lineage and that the SBD/KR/KNU SYG06/06 isolate was clearly different from HPAI H5N1 strains, including human isolates and the Italian HPAI H5N2 strains. Additionally, no relationship was found between SBD/KR/KNU SYG06/06 and the Korean HPAI H5N1 isolates. The SBD/KR/ KNU SYG06/06 isolate had avian specific receptor binding site residues in the HA protein and the four C-terminal amino acids in the NS1 protein. The HA protein of the SBD/KR/KNU SYG06/06 isolate exhibited the typical LPAI motif at the cleavage site and this virus produced no cytopathic effects in MDCK cells without trypsin. Given these results, we suggest that the H5N3 AIV isolated from the spot-billed duck should be considered an LPAI virus and should have no pathogenic effect in humans.
\end{abstract}

(Key words : Avian influenza virus, H5N3, Phylogenetic analysis, Spot-billed duck)

\section{INTRODUCTION}

Influenza A viruses are negative-sense RNA viruses whose genomes are divided into eight segments that code for 11 proteins (Chen et al., 2001). The viruses have been classified into subtypes based on their surface glycoproteins, hemagglutinin (HA) and neuraminidase (NA). There are 16 known HA subtypes (H1 through H16) and 9 known NA subtypes (N1 through N9) of type A influenza viruses (Fouchier et al., 2005). Avian influenza viruses (AIVs) are categorized into two pathotypes based on their virulence: low pathogenic avian influenza (LPAI) virus and highly pathogenic avian influenza (HPAI) virus. Among the $16 \mathrm{HA}$ subtypes of AIV, only the H5 and H7 subtypes have caused HPAI in poultry. However, most H5 or H7 subtype viruses are LPAI viruses. The HA molecules of HPAI viruses differ from those of LPAI viruses in that they possess multiple basic amino acids at the carboxyl terminus of HA1. Basic amino acids adjacent to the cleavage site allow ubiquitous intracellular proteases other than trypsin-like proteases to cleave the HA into HAl and HA2 domains. This enables the virus to cause systemic infec- tion with high mortality in poultry (Stieneke-Gröber et al., 1992; Seo et al., 2002). Previous studies have demonstrated that some LPAI viruses of the $\mathrm{H} 5$ and $\mathrm{H} 7$ subtypes can mutate into HPAI viruses, and several mechanisms involved in the emergence of HPAI virus from a LPAI virus precursor have been documented (Banks et al., 2001; Duan et al., 2007; Horimoto et al., 1995). For example, an LPAI virus evolved into an HPAI virus that caused a severe outbreak in Pennsylvania in 1983 (Kawaoka et al., 1984). In addition, a study conducted in northern Europe suggested that the LPAI subtypes of the $\mathrm{H} 5$ and $\mathrm{H} 7$ viruses resident in a migratory duck were introduced to poultry, causing HPAI H5N2 virus and H7N7 outbreaks (Munster et al., 2005). Since the 1997 H5N1 avian influenza outbreak in Hong Kong (Shortridge et al., 1998), avian influenza viruses, including the $\mathrm{H} 5$ and $\mathrm{H} 7$ HPAI viruses, have demonstrated the ability to infect humans directly.

The first HPAI outbreak in South Korea's history was the H5N1 HPAI outbreak of chickens in South Korea in December 2003 (Lee et al., 2005). Although there have been several subsequent outbreaks of H5N1 HPAI since the 2003 HPAI

\footnotetext{
${ }^{\dagger}$ To whom correspondence should be addressed : sunghw@kangwon.ac.kr
} 
outbreak, no human cases have been identified in Korea. This study describes the biological and molecular characterization of an H5N3 AIV isolated from spot-billed duck (Anas poecilorhyncha) Additionally, we genetically characterized the H5N3 isolate and compared the virus with AIVs, including HPAI H5N1, from other countries. This study will provide an understanding of the origin and lineage of the H5N3 virus and its potential pathogenicity.

\section{MATERIALS AND METHODS}

\section{Virus Isolation}

An avian H5N3 influenza virus (A/spot-billed duck/Korea/ KNU SYG06/2006(H5N3)) was isolated from fecal samples of spot-billed duck (Anas poecilorhyncha) obtained from SoYang river in Korea. We identified bird species with mitochondrial DNA barcoding method. DNA was extracted from fecal samples and was amplified with mitochondrial specific primers. DNA sequences of PCR products were compared with the sequence database offered by the Barcode of Life Data Systems (http://www.boldsystems.org/views/login.php).

Fecal samples obtained from spot-billed duck were suspended in phosphate buffered saline (PBS) containing antibiotic solution (100 unit/ $/ \mu \mathrm{L}$ of penicillin and $100 \mathrm{mg} / \mathrm{\mu L}$ of strep-to mycin) and thoroughly mixed by vortexing. The mixture was centrifuged at 3,000 rpm for $10 \mathrm{~min}$ and supernatants were filtered with $0.45 \mu \mathrm{m}$ Millex syringe-driven filters (Millipore, USA). The filtered aqueous solution was inoculated in 10day-old embryonating chicken eggs (ECEs) via the allantoic sac. After $3 \sim 5$ days of incubation, the allantoic fluid from inoculated eggs was harvested. The presence of AI virus was determined by hemagglutination assay (HA assay) performed according to the World Organization for Animal Health recommendations (http://www.oie.int/eng/normes/mmanual/A_summry. $\mathrm{htm}$ ). AI virus was subtyped by reverse transcription polymerase chain reaction (RT-PCR) assays as described previously (Qiu et al., 2009; Tsukamoto et al., 2008) and confirmed by sequencing.

\section{Reverse Transcription (RT)-PCR and Sequencing}

Viral RNA was extracted directly from allantoic fluid from ECEs using a Viral Gene-spin viral DNA/RNA extraction kit
(iNtRON Biotechnology, Korea) according to the manufacturer's instructions. The entire coding regions of all eight genes of the influenza virus were amplified by standard RT-PCR with the Qiagen one-step RT-PCR kit (QIAGEN, USA). The RT-PCR conditions and segment-specific primer sequences have been previously described (Hoffmann et al., 2001; Li et al., 2007). The PCR products were separated by agarose gel electrophoresis, and amplicons with the appropriate sizes were subsequently excised from the gel. The DNA fragments were extracted and purified using a QIAquick gel extraction kit (QIAGEN, USA). Sequencing was performed with an ABI 373 XL DNA sequencer (Applied Biosystems, USA) at Macrogen Inc (Macrogen, Korea).

\section{Genetic and Phylogenetic Analysis}

Nucleotide BLASTn analysis (http://www.ncbi.nlm.nih.gov/ BLAST) was used to identify related genes of the virus and the reference sequences were obtained from GenBank. Nucleotide and amino acid sequences obtained in this study were aligned by the Clustal W Method and edited with MEGA version 4.0 software. Pair-wise distance calculation was also performed with the MEGA version 4.0 software to determine nucleotide and amino acid sequence similarity. Phylogenetic trees were constructed together with selected strains available in GenBank. Phylogenetic relationships of the aligned sequences for eight gene segments were estimated by neighbor-joining method with 500 bootsrap replicates with the MEGA version 4.0 software (Tamura et al., 2007).

\section{Replication in MDCK Cells}

Cytopathic effect (CPE) of the virus was examined in Madin-Darby canine kidney (MDCK) cells with or without trypsin according to the WHO manual on animal influenza diagnosis and surveillance (http://www.who.int/csr/resources/ publications/influenza/whocdscsrncs20025.p). Cell cultures were harvested when more than $75 \%$ of the total cells in the monolayer showed CPE. The cell culture supernatants were tested by HA assay with chicken erythrocytes.

\section{Nucleotide Sequence Accession Numbers.}

All of the sequence data for the SBD/KR/KNU SYG06/06 (H5N3) virus reported herein were deposited to the GenBank 
database under the accession numbers JF800144 JF800151.

\section{RESULTS}

\section{Sequence and Phylogenetic Analysis}

The sequences of the whole genome as well as the amino acids of all eight segments were determined to characterize the $\mathrm{SBD} / \mathrm{KR} / \mathrm{KNU} \mathrm{SYG06/06}$ virus. A phylogenetic analysis of the eight viral genes (Fig. $1 \mathrm{a} \sim \mathrm{h}$ ) revealed that all segments belonged to the Eurasian avian lineage. The percent similarities among the eight gene segments of the $\mathrm{SBD} / \mathrm{KR} /$ KNU SYG06/06 isolate with Eurasian avian viruses ranged from $98.2 \sim 99.8 \%$ for nucleotide sequences and $98.3 \sim 100 \%$ for amino acid sequences (Table 1).

\section{HA Gene}

A phylogenetic analysis of the H5 gene revealed that the $\mathrm{SBD} / \mathrm{KR} / \mathrm{KNU}$ SYG06/06 viruses separated into Eurasian lineages. Within the Eurasian lineage, at least two distinct clusters were observed (Fig. 1a). One cluster (HPAI) contained the "HP H5N1" group of viruses, including human isolates as well as the "HP H5N2" viruses obtained from Italy from 1997 to 1998, while the other cluster contained the LPAI viruses. The $\mathrm{SBD} / \mathrm{KR} / \mathrm{KNU} \mathrm{SYG06/06}$ isolate belonged to the LPAI subgroup. Phylogenetically, the South Korean HPAI H5N1 stains isolated in 2003 and 2006 were clearly different from the $\mathrm{SBD} / \mathrm{KR} / \mathrm{KNU} \mathrm{SYG06/06}$ isolate, and they belonged to the "HP H5N1" subgroup.

The SBD/KR/KNU SYG06/06 isolate shared the highest sequence identity with the A/aquatic bird/Korea/w54/2005 (H5N2) isolate, with 99.2\% nucleotide and 99.3\% amino acid sequence identities (Table 1).

\section{NA Gene}

A phylogenetic analysis of the N3 gene showed that the NA gene from the SBD/KR/KNU SYG06/06 isolate also belong ed to the Eurasian avian lineage (Fig. 1b). In addition, the N3 gene of the $\mathrm{SBD} / \mathrm{KR} / \mathrm{KNU} \mathrm{SYG06/06}$ isolate belonged to the "Recent N3" subgroup and was closely related to H5N3 viruses recently isolated in China and Japan. The N3 gene of the SBD/KR/KNU SYG06/06 isolate shared 98.2\% nucleotide identity and 98.3\% amino acid identity with A/ duck/Chiba/ 1/2006 (H5N3) (Table 1).

\section{Internal Genes}

The phylogenetic tree for the other six internal genes (NP, NS, M, PA, PB1, and PB2) showed similar clustering in the Eurasian avian lineage and exhibited no close relationships with HPAI viruses (Fig. 1c $\sim \mathrm{h}$ ). The phylogenetic tree based on the NS gene readily identified the two previously described subtypes (A and B) of the NS gene. The NS gene of the SBD/ $\mathrm{KR} / \mathrm{KNU}$ SYG06/06 isolate, along with Hong Kong H5N1, Italy H5N2, and South Korea H5N1 highly pathogenic viruses, belonged to subgroup $\mathrm{A}$, whereas the Italy $\mathrm{H} 5 \mathrm{~N} 3$ viruses isolated in 2002 and China H5N3 and H5N2 viruses isolated in 2005 belonged to subgroup B (Fig. 1c). The phylogenetic trees of the M, PB1, and PB2 genes showed two distinct clusters within the Eurasian lineage, HPAI and

Table 1. Genetic similarity among eight gene segments of SBD/KR/KNUSYG06/06 and other influenza isolates

\begin{tabular}{clcc}
\hline \hline \multirow{2}{*}{ Gene } & \multicolumn{1}{c}{ Virus with highest identity } & \multicolumn{2}{c}{ Identity(\%) $^{\text {aa }^{\mathrm{b}}}$} \\
\cline { 3 - 4 } HA & A/aquatic bird/Korea/w54/2005(H5N2) & nt $^{\mathrm{a}}$ & 99.3 \\
NA & A/duck/Chiba/1/2006(H5N3) & 99.2 & 98.3 \\
NP & A/mallard/Jiangxi/12147/2005(H6N2) & 99.4 & 99.6 \\
NS & A/aquatic bird/India/NIV-17095/2007(H11N1) & 98.7 & 100 \\
M & A/gull/Astrakhan/1846/1998(H13N6) & 99.3 & 99.9 \\
PA & A/mallard/Jiangxi/8264/2004(H6N2) & 99.3 & 99.5 \\
PB1 & A/mute swan/Aktau/1460/2006(H5N1) & 98.3 & 99.7 \\
PB2 & A/duck/Primorie/2633/2001(H5N3) & 98.5 & 9 \\
\hline
\end{tabular}

\footnotetext{
${ }^{\mathrm{a}} \mathrm{nt}$ : Nucleotide, ${ }^{\mathrm{b}}$ aa : Amino acid.
} 
(a) HA

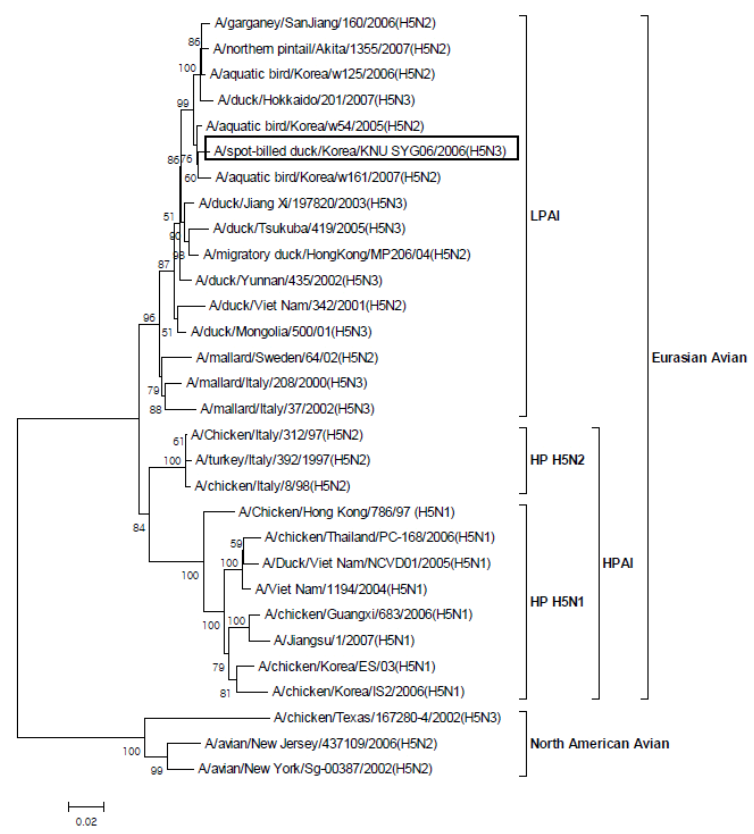

(c) NS

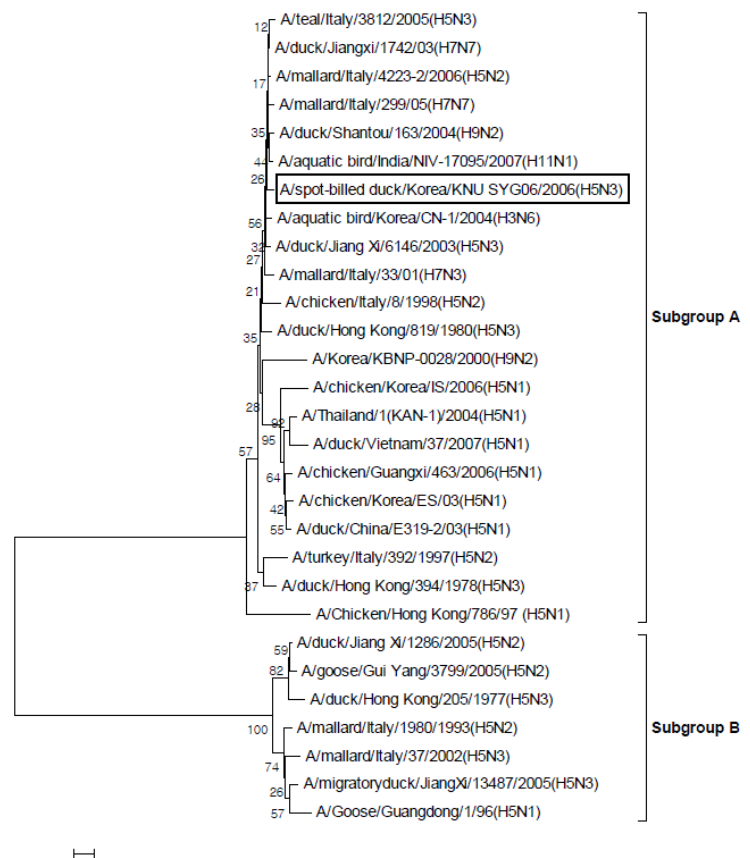

(b) NA

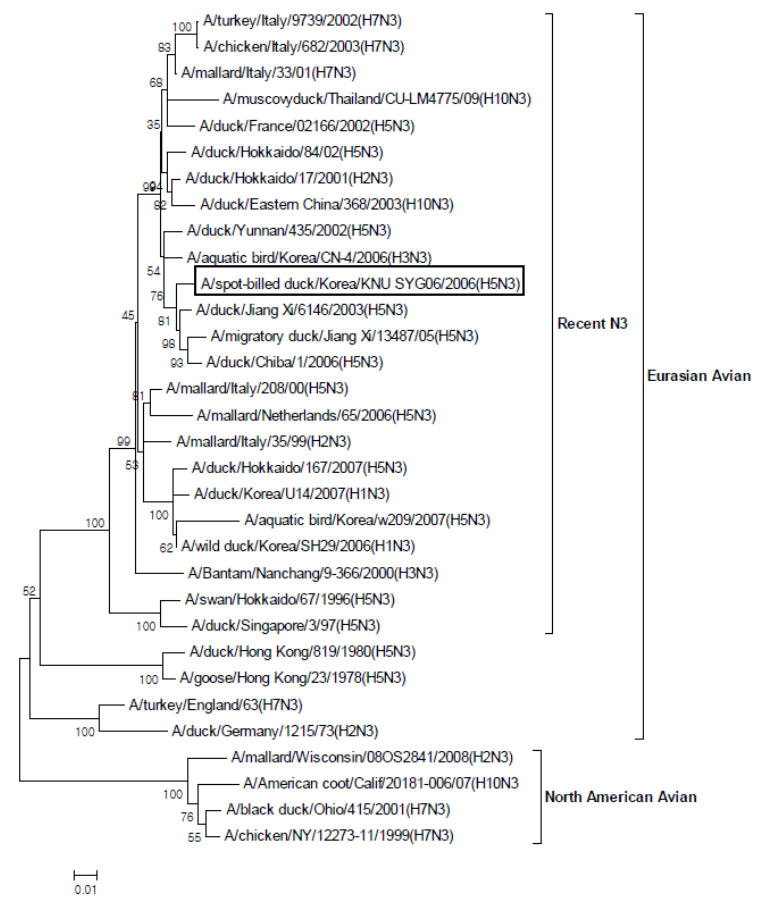

(d) NP

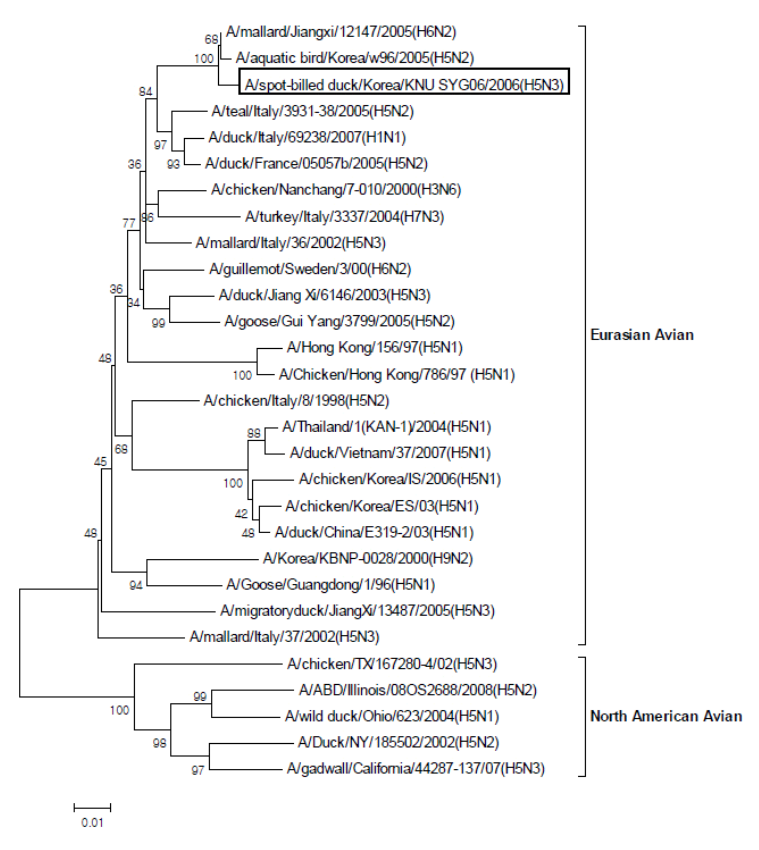

Fig. 1. Phylogenetic trees based on nucleotide sequences of HA (a), NA (b), NP (c), NS (d), M (e), PA (f), PB1 (g), PB2 (h) genes. The tree was generated by neighbour-joining method with MEGA 4.0 program, using bootstrap replication (500 bootstraps). An $\mathrm{H} 5 \mathrm{~N} 3$ isolate $(\mathrm{SBD} / \mathrm{KR} / \mathrm{KNU} \mathrm{SYG06/06)}$ in this study was boxed. 
(e) $M$

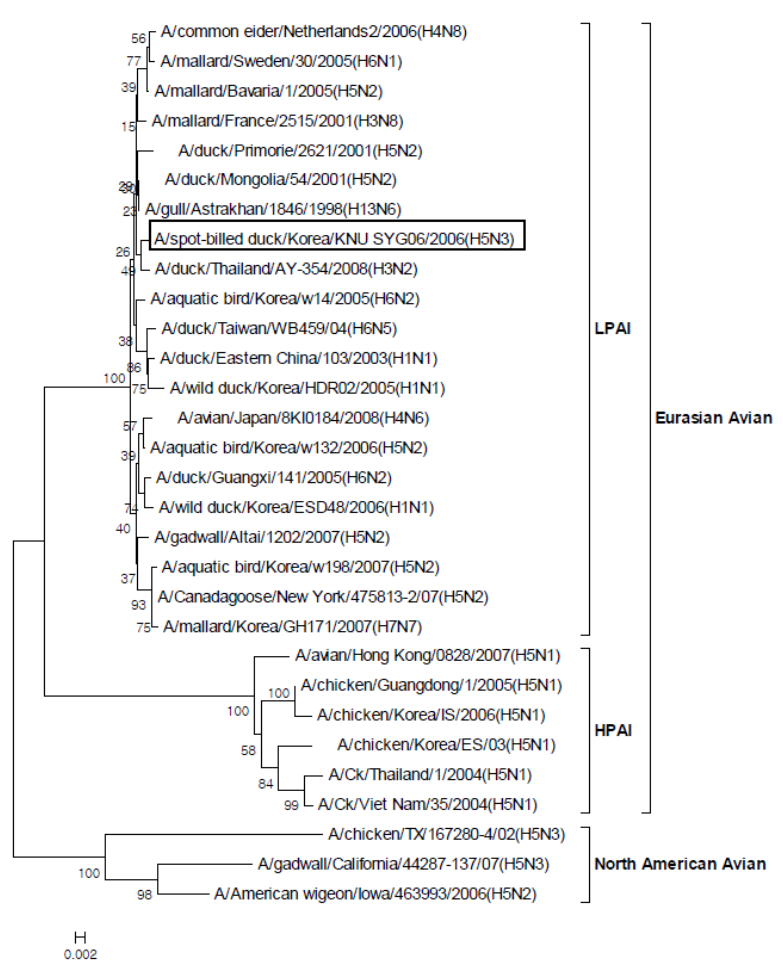

(g) PB1

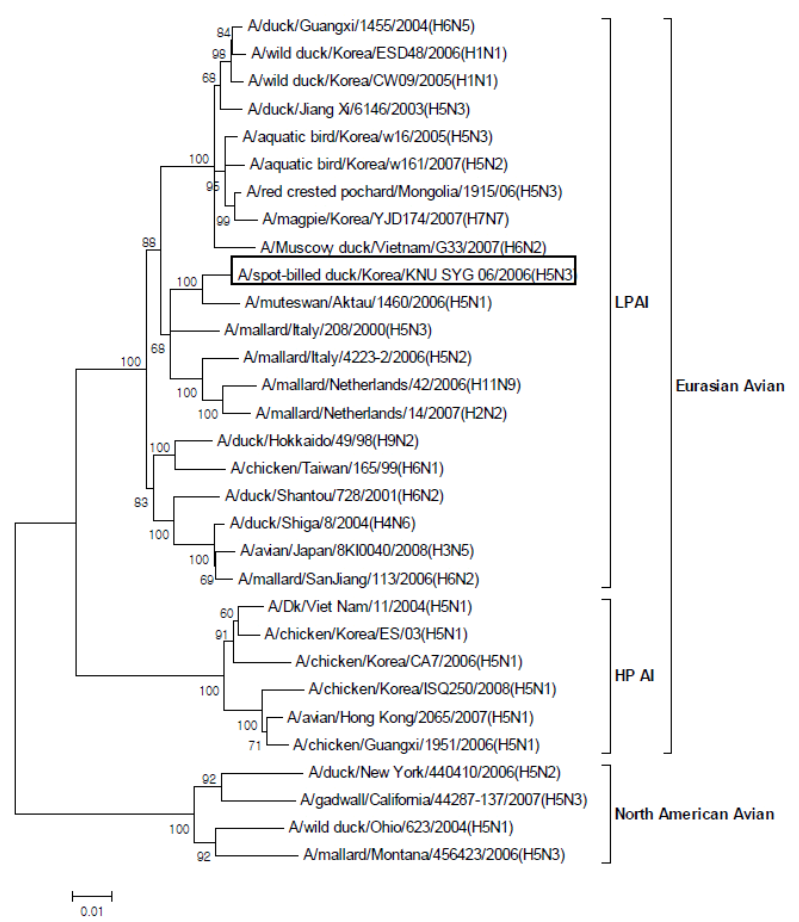

(f) PA

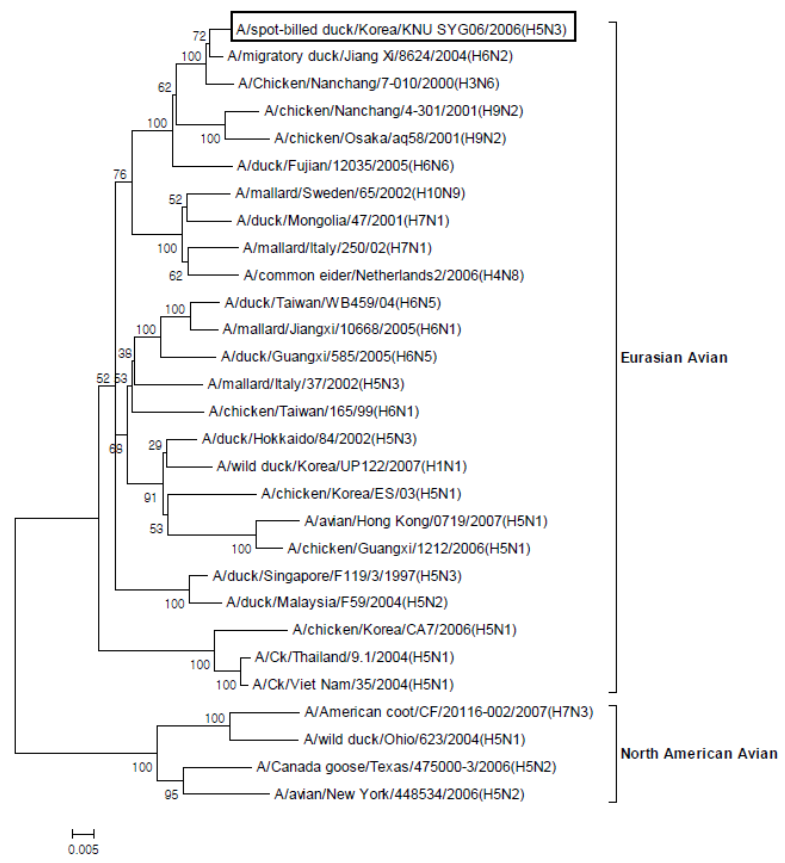

(h) PB2

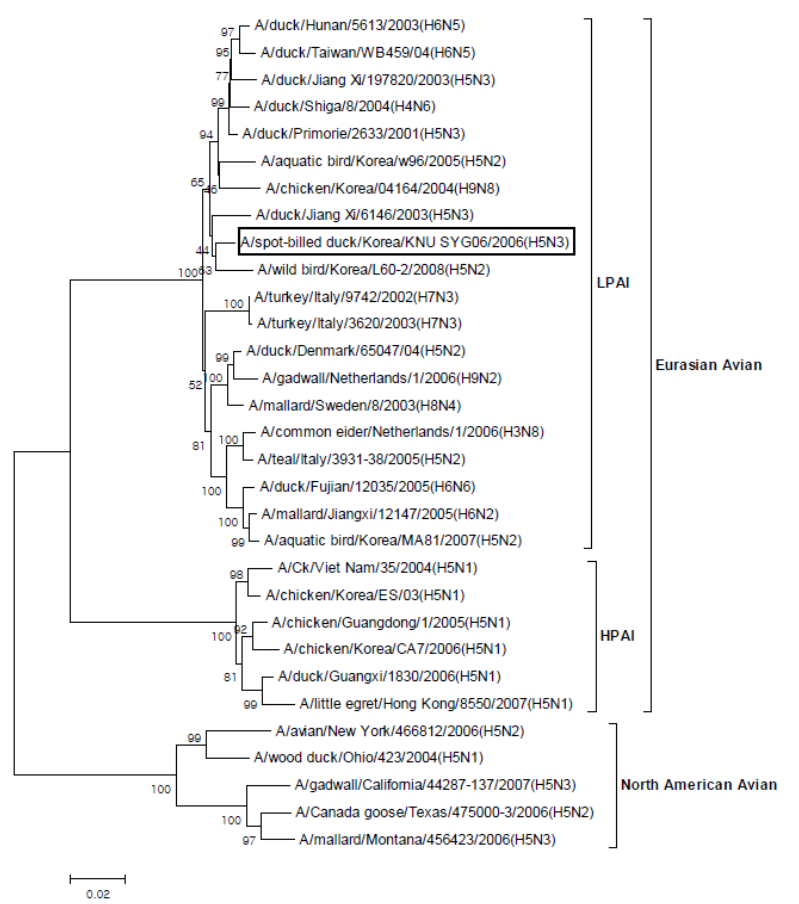

Fig. 1. Continued. 
LPAI. The SBD/KR/KNU SYG06/06 isolate belonged to the LPAI group.

The highest identities of nucleotide and amino acid sequences are indicated in Table 1. The PB1 gene shared the highest sequence identity with the low pathogenic $\mathrm{A} /$ mute swan/Aktau/1460/2006(H5N1) isolate.

\section{Molecular Characterization}

Based on the amino acid sequence at the HA1-HA2 connecting peptide, the $\mathrm{SBD} / \mathrm{KR} / \mathrm{KNU} \mathrm{SYG06/06}$ isolate exhibited a RETR/GLF motif. (Table 2). All isolates analyzed in this study exhibited the conserved amino acid residues at positions 150, 202, 206, 237, 238, and 240 (position 138, 190, 194, 225, 226, and 228 in H3 numbering), which define the receptor binding site and are related to the preferential binding of sialic acids joined to the sugar chain through an a-2,3 linkage. (Table 2) (Matrosovich et al., 1997).

The N3 gene was compared with other available N3 sequences with differing HA subtypes. We observed that the Italy H7N3 isolates from 2002 to 2003 had a 23 amino acid deletion in the stalk region (position 56 to 78 ) that was not found in other Eurasian N3 genes, including the SBD/KR/ KNU SYG06/06 isolate (Table 3).

The four C-terminal amino acids of the NS1 gene bind to the protein interaction domain and perform localized signaling at particular subcellular locations (Obenauer et al., 2006). The $\mathrm{SBD} / \mathrm{KR} / \mathrm{KNU}$ SYG06/06 isolate analyzed in this study exhibited the C-terminal motifs, ESEV. Within the NS1 gene, amino acid changes at position 149 (V149A) have been associated with virulence and resistance to antiviral cytokines in chickens ( $\mathrm{Li}$ et al., 2006). The $\mathrm{SBD} / \mathrm{KR} / \mathrm{KNU} \mathrm{SYG06/06}$ isolate had 149A.

Within the PB2 protein, the mutations E627K and D701N have previously been identified as determinants of virulence in mammalian hosts (Steel et al., 2009). These mutations were absent in the $\mathrm{SBD} / \mathrm{KR} / \mathrm{KNU} \mathrm{SYG06/06}$ isolate.

The NA protein of the SBD/KR/KNU SYG06/06 isolate possessed residue H276, which is a known marker for sensitivity to the neuraminidase inhibitor oseltamivir (Hayden, 2006). The amino acid sequence analysis of the M2 protein revealed that the SBD/KR/KNU SYG06/06 isolate contained no mutations responsible for resistance to amantadine, the M2 inhibitor (Cheung et al., 2006).

\section{Replication in MDCK Cells}

We detected the virus titers with HA reaction after MDCK cells were inoculated with the $\mathrm{SBD} / \mathrm{KR} / \mathrm{KNU} \mathrm{SYG06/06}$ isolate. The HA activity of the virus was observed in MDCK cells with the addition of trypsin; however, there were no detectable viruses in MDCK cultures without trypsin.

Table 2. Comparison of amino acid sequence of different H5 viruses

\begin{tabular}{|c|c|c|c|c|c|c|c|}
\hline \multirow{2}{*}{ Strain } & \multirow{2}{*}{$\begin{array}{l}\text { HA cleavage site sequence at } \\
\text { amino acid } 339-349\end{array}$} & \multicolumn{6}{|c|}{ Conserved amino acid in $\mathrm{RBS}^{\mathrm{b}}$} \\
\hline & & 150 & 202 & 206 & 237 & 238 & 240 \\
\hline \multicolumn{8}{|l|}{ Low pathogenic } \\
\hline SBD/Korea/KNU SYG06/06 (H5N3) ${ }^{a}$ & 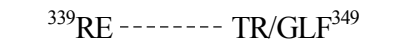 & $\mathrm{A}$ & E & $\mathrm{L}$ & G & $\mathrm{Q}$ & $\mathrm{G}$ \\
\hline Aquatic bird/Korea/w54/05 (H5N2) & RE ------ TR/GLF & A & $\mathrm{E}$ & $\mathrm{L}$ & G & Q & G \\
\hline Mallard/Italy/36/2002 (H5N3) & RE------ TR/GLF & A & $\mathrm{E}$ & $\mathrm{L}$ & G & Q & G \\
\hline Mallard/JiangXi/13487/05 (H5N3) & RE ------ TR/GLF & A & $\mathrm{E}$ & $\mathrm{L}$ & G & Q & G \\
\hline \multicolumn{8}{|l|}{ Highly pathogenic } \\
\hline Chicken/Italy/8/98 (H5N2) & RRRK ----- KR/GLF & A & $\mathrm{E}$ & $\mathrm{L}$ & G & Q & G \\
\hline Hong Kong/481/97 (H5N1) & RERRRK - KR/GLF & $\mathrm{A}$ & $\mathrm{E}$ & $\mathrm{L}$ & G & Q & G \\
\hline Chicken/Hong Kong/786/97 (H5N1) & REKRKK - KR/GLF & $\mathrm{A}$ & E & $\mathrm{L}$ & G & Q & G \\
\hline Chicken/Korea/ES/03 (H5N1) & RERRR - KR/GLF & A & $\mathrm{E}$ & $\mathrm{L}$ & G & Q & G \\
\hline Chicken/Korea/IS2/06 (H5N1) & GERRRK - KR/GLF & $\mathrm{A}$ & $\mathrm{E}$ & $\mathrm{L}$ & G & Q & G \\
\hline Chicken/Guangxi/683/06 (H5N1) & RERRR - KR/GLF & A & E & $\mathrm{L}$ & G & Q & G \\
\hline
\end{tabular}

a The isolate in boldface is the $\mathrm{SBD} / \mathrm{KR} / \mathrm{KNU}$ SYG06/06 (H5N3) isolate analyzed in this study.

${ }^{\mathrm{b}}$ RBS : receptor binding site. 
Table 3. Aligment of the NA stalk region of N3 viruses

\begin{tabular}{|c|c|c|}
\hline Strain & Subetype & Amino acid sequence from 40 to 91 \\
\hline $\mathrm{SBD} / \mathrm{Korea} / \mathrm{KNU} \mathrm{SYG} 06 / 06^{\mathrm{a}}$ & H5 & GDHQIVVYPTTTAPVVPNCSDTIITYNNTVVNNITTTIITKEETHFKSSLPL \\
\hline Duck/Jiang Xi/6146/03 & H5 & GDHQTVVYPTTTAPVVPNCSDTIITYNNTVVNNITTTIITKEETHFKSSLPL \\
\hline Mallard/Italy/208/00 & H5 & GDHQTVVYPTITAPVVPNCSDTIITYNNTVVNNITTTIITKAEKHFKSSLPL \\
\hline Mallard/Italy/33/01 & $\mathrm{H} 7$ & GDHQTVVYPTVTAPVVPNCSDTIITYNNTVVNNITTTIVTKAETHFKSSLPL \\
\hline Turkey/Italy/214845/02 & $\mathrm{H} 7$ & GDHQTVVYPTVTAPVV -------. \\
\hline Turkey/Italy/9739/02 & $\mathrm{H} 7$ & GDHQTVVYPTVTAPVV - \\
\hline Tukey/Italy/3620/03 & $\mathrm{H} 7$ & GDHQTVVYPTVTAPVV ----- \\
\hline
\end{tabular}

a The isolate in boldface is the $\mathrm{SBD} / \mathrm{Korea} / \mathrm{KNU} \mathrm{SYG06/06}(\mathrm{H} 5 \mathrm{~N} 3)$ isolate analyzed in this study.

\section{DISCUSSION}

We analyzed the genetic and biologic characteristics of the $\mathrm{H} 5 \mathrm{~N} 3$ virus $(\mathrm{SBD} / \mathrm{KR} / \mathrm{KNU} \mathrm{SYG06/06)}$ isolated from spotbilled duck fecal samples. Our phylogenetic analysis showed that the $\mathrm{SBD} / \mathrm{KR} / \mathrm{KNU} \mathrm{SYG06/06}$ viruses were closely related to LPAI viruses isolated in Europe and Asia. However, the $\mathrm{SBD} / \mathrm{KR} / \mathrm{KNU} \mathrm{SYG06/06}$ isolate was not closely related to HPAI strains, including the South Korean HPAI strains. The genetic similarity at the nucleotide and amino acid sequence levels suggested that all eight gene segments of the $\mathrm{SBD} / \mathrm{KR} /$ KNU SYG06/06 isolate shared the highest sequence identity with Eurasian avian influenza viruses, particularly LPAI viruses from different countries isolated in different years. This result provides evidence of segment reassortment among different influenza A viruses.

To determine the pathogenic potential of the SBD/KR/KNU SYG06/06 isolate, we analyzed its biological and genetic characteristics. The results showed that the $\mathrm{SBD} / \mathrm{KR} / \mathrm{KNU} \mathrm{SYG06/}$ 06 isolate was a low-pathogenicity AIV. Firstly, the H5 gene had a RETR/GLF sequence at the cleavage site, suggesting the low pathogenicity of the AIVs. Secondly, the MDCK cell-culture system appeared to lack CPEs and no replication of viruses was observed in the absence of added trypsin.

Several low-pathogenicity AIVs of the H5 and H7 subtypes have been shown to undergo a virulence shift to high pathogenicity either in the field or in laboratory test systems. Among the $\mathrm{H} 5$ and $\mathrm{H} 7$ influenza viruses, however, we cannot predict which low-pathogenicity AIVs will retain low pathogenicity and which will mutate to become highly pathogenic. In addition, the type of mutation that is necessary to increase the virulence is not predictable. What has been observed is that some H5 and H7 subtype AIVs, when allowed to circulate in poultry for an extended period of time, can undergo mutational changes that can have serious consequences.

A deletion of variable length in the NA stalk region is a molecular feature frequently found in viruses isolated from domestic poultry. This change represents an adaptation of a wild bird virus to a new host, such as domestic poultry (Matrosovich et al., 1995). The $\mathrm{SBD} / \mathrm{KR} / \mathrm{KNU} \mathrm{SYG06/06}$ isolate did not exhibit the NA stalk deletion. However, the Italian H7N3 isolated from turkey during 2002 2003 exhibited a 23 amino acid deletion in the stalk region (Campitelli et al., 2004). Previous studies have demonstrated that a deletion in the NA gene decreases the ability of the virus to be released from host cells. To compensate for the decreased NA activity for efficient replication of the virus, the HA activity of the cell receptor can also be decreased. This can be achieved by acquiring additional carbohydrates at the HA head, which can decrease the affinity of the receptor binding site in the HA to the cell receptor by steric hindrance (Klenk et al., 2002).

Differences in the receptor-binding sites between human and avian viruses represent a major barrier to efficient transmission of avian viruses to humans (Matrosovich et al 1997). Moreover, as little as two amino acid substitutions may be sufficient to cause a change in receptor-binding preference from the avian $a-2,3$ to the $a-2,6$ sialic acid receptor, as shown for the H5N1 and H1N1 subtype viruses (Yamad et al., 2006; Tumpey et al., 2007). Our study showed that the $\mathrm{SBD} / \mathrm{KR} / \mathrm{KNU} \mathrm{SYG06/06}$ isolate had the avian specific conserved sequence.

A large-scale sequence analysis of avian influenza viruses 
indicated that the four C-terminal residues of the NS1 protein form a PDZ domain ligand (Obenauer et al., 2006). PDZ domains are protein-protein recognition modules that organize diverse cell-signaling assemblies and perform localized signaling at particular subcellular locations. Proteins that contain PDZ domains play important roles in many key signaling pathways, including regulating the activity and trafficking of membrane proteins, and maintain cell polarity and morphology. Of the human influenza viruses, $90 \%$ have a NS1 protein with a C-terminal motif sequence of RSKV or RSEV. However, $81 \%$ of avian origin viruses have a NS1 protein with a Cterminal motif sequence of ESEV or EPEV (Obenauer et al., 2006). The $\mathrm{SBD} / \mathrm{KR} / \mathrm{KNU} \mathrm{SYG06/06}$ isolate exhibited the C-terminal motif, ESEV.

Several amino acid mutations of the PB2 protein may have important effects on the virulence and adaptation of the influenza virus in mammalian hosts. One of the most commonly identified virulence markers to date is an amino acid change at E627K in the PB2 protein. In the HPAI H5N1 and H7N7 viruses, E627K was shown to be the prime determinant of pathogenesis in mice (Steel et al., 2009; Chen et al., 2006). The D701N mutation in the PB2 protein has previously been shown to expand the host range of avian H5N1 to mice and humans ( $\mathrm{Li}$ et al., 2005). These two point mutations were not present in the $\mathrm{SBD} / \mathrm{KR} / \mathrm{KNU} \mathrm{SYG06/06}$ isolate.

Currently available anti-influenza drugs include the adamantanes (amantadine and rimantadine) and the neuraminidase inhibitors oseltamivir and zanamivir. Mutations in the M2 and NA proteins that are known to confer resistance to anti-influenza drugs were not found in the $\mathrm{SBD} / \mathrm{KR} / \mathrm{KNU}$ SYG06/06 isolate. Mutated viruses can reportedly either lose the ability to bind M2 ion channel blockers or can bind the drug, but retain M2 functioning (Wang et al., 1993). Although amantadine-resistant $\mathrm{H} 5 \mathrm{~N} 1$ viruses are present in Asia, their distribution appears to be largely limited to Thailand and Vietnam (Cheung et al., 2006). It is perhaps surprising that most H5N1 isolates from China and Indonesia are sensitive to amantadine given the reportedly widespread administration of amantadine to farmed poultry in those countries. The apparent geographical disparity in the resistance of $\mathrm{H} 5 \mathrm{~N} 1$ isolates to amantadine is unexplained. No known resistance-linked mutation was identified among isolated H5N1 viruses from
Japan and Korea from 1996 to 2005 (Cheung et al., 2006).

Lee et al. (2012) reported that four LPAI H5N2 AIVs isolated from wild bird in Korea could not be replicated ant transmitted in chickens. The $\mathrm{H} 5$ gene of the SBD/KR/KNU SYG06/06 isolate had a same sequence (RETR/GLF) with LPAI H5 isolates which Lee et al. reported. This result suggested that the SBD/KR/KNU SYG06/06 isolate should be categorized as an LPAI virus. However, LP H5 viruses have the potential to evolve into HPAI viruses when circula- ting in terrestrial poultry for prolonged periods. To avoid serious future outbreaks, continued systematic influenza surveillance in both domestic and migratory birds in Korea is necessary, and additional understanding of the $\mathrm{H} 5 \mathrm{~N} 1$ viruses from different countries is required.

\section{CONCLUSION}

A phylogenetic analysis of the eight viral genes showed that an $\mathrm{H} 5 \mathrm{~N} 3$ avian influenza virus $(\mathrm{SBD} / \mathrm{KR} / \mathrm{KNU}$ SYG06/06) isolated from spot-billed duck belongs to the Eurasian lineage and that the isolate was clearly different from HPAI H5N1 strains. The SBD/KR/KNU SYG06/06 isolate had avian specific receptor binding site residues in the $\mathrm{HA}$ protein and the four C-terminal amino acids in the NS1 protein. The HA protein of the SBD/KR/KNU SYG06/06 isolate exhibited the typical LPAI motif at the cleavage site and this virus produced no cytopathic effects in MDCK cells without trypsin. Given these results, we suggest that the H5N3 avian influenza virus isolated from the spot-billed duck should be considered an LPAI virus and should have no pathogenic effect in humans.

\section{ACKNOWLEDGEMENTS}

This research was supported by the Happy tech. program through the National Research Foundation of Korea (NRF) funded by the Ministry of Education, Science and Technology (grant No. 2010-0020802)

\section{REFERENCES}

Banks J, Speidel ES, Moore E, Plowright L, Piccirillo A, 
Capua I, Cordioli P, Fioretti A, Alexander DJ 2001 Changes in the haemagglutinin and the neuraminidase genes prior to the emergence of highly pathogenic H7N1 avian influenza viruses in Italy. Arch Virol 146:963-973.

Campitelli L, Mogavero E, De Marco MA, Delogu M, Puzelli S, Frezza F, Facchini M, Chiapponi C, Foni E, Cordioli P, Webby R, Barigazzi G, Webster RG, Donatelli I 2004 Interspecies transmission of an H7N3 influenza virus from wild birds to intensively reared domestic poultry in Italy. Virology 323:24-36.

Chen GW, Chang SC, Mok CK, Lo YL, Kung YN, Huang JH, Shih YH, Wang JY, Chiang C, Chen CJ, Shih SR 2006 Genomic signatures of human versus avian influenza A viruses. Emerg Infect Dis 12:1353-1360.

Chen W, Calvo PA, Malide D, Gibbs J, Schubert U, Bacik I, Basta S, O'Neill R, Schickli J, Palese P, Henklein P, Bennink JR, Yewdell JW 2001 A novel influenza A virus mitochondrial protein that induces cell death. Nat Med 7:1306-1312.

Cheung CL, Rayner JM, Smith GL, Wang P, Naipospos TS, Zhang J, Yuen KY, Webster RG, Peiris JS, Guan Y, Chen H 2006 Distribution of amantadine-resistant H5N1 avian influenza variants in Asia. J Infect Dis 193:1626-1629.

Duan L, Campitelli L, Fan XH, Leung YH, Vijaykrishna D, Zhang JX. Donatelli I, Delogu M, Li KS, Foni E, Chiapponi C, Wu WL, Kai H, Webster RG, Shortridge KF, Peiris JS, Smith GJ, Chen H, Guan Y 2007 Characterizaion of low-pathogenic H5 subtype influenza viruses from Eurasia: Implications for the origin of highly pathogenic H5N1 viruses. J Virol 81:7529-739.

Fouchier RA, Munster V, Wallensten A, Bestebroer TM, Herfst S, Smith D, Rimmelzwaan GF, Olsen B, OsterhausAD 2005 Characterization of a novel influenza A virus hemagglutinin subtype (H16) obtained from black-headed gulls. J Virol 79:2814-2822.

Hayden FG 2006 Antivirals for influenza: historical perspectives and lessons learned. Antiviral Res 71:372-378.

Hoffmann E, Stech J, Guan Y, Webwter RG, Perez DR 2001 Universal primer set for the full-length amplification of all influenza A viruses. Arch Virol 146:2275-2289.

Horimoto T, Rivera E, Pearson J, Senne D, Krauss S, Kawaoka Y, Webster RG 1995 Origin and molecular changes associated with emergence of a highly pathogenic H5N2 influenza virus in Mexico. Virology 213:223-230.

Kawaoka Y, Naeve CW, Webster RG 1984 Is virulence of H5N2 influenza viruses in chickens associated with loss of carbohydrate from the hemagglutinin? Virology 139(2): 303-316.

Klenk HD, Wagner R, Heuer D, Wolff T 2002 Importance of hemagglutinin glycosylation for the biological functions of influenza virus. Virus Res 82:73-75.

Lee CW, Suarez DL, Tumpey TM, Sung HW, Kwon YK, Lee YJ, Choi JG, Joh SJ. Kim MC, Lee EK, Park JM, Lu X, Katz JM, Spackman E, Swayne DE, Kim JH 2005 Characterization of highly pathogenic H5N1 avian influenza A viruses isolated from South Korea. J Virol 79: 3692-3702.

Lee DH, Kwon JH, Park JK, Lee YN, Yuk SS, Lee JB, Park SY, Choi IS, Song CS 2012 Characterization of lowpathogenicity $\mathrm{H} 5$ and $\mathrm{H} 7$ Korean avian influenza viruses in chickens. Poultry Sci 91:3086-3090.

Li OT, Barr I, Leung CY, Chen H, Guan Y, Peiris JS, Poon LL 2007 Reliable universal RT-PCR assays for studying influenza polymerase subunit gene sequences from all 16 haemagglutinin subtypes. J Virol Methods 142:218-222.

Li Z, Chen H, Jiao P, Deng G, Tian G, Li Y, Hoffmann E, Webster RG, Matsuoka Y, Yu K 2005 Molecular basis of replication of duck H5N1 influenza viruses in a mammalian mouse model. J Virol 79: 12058-12064.

Li Z, Jiang Y, Jiao P, Wang A, Zhao F, Tian G, Wang X, Yu K, Bu Z, Chen H 2006 The NS1 gene contributes to the virulence of $\mathrm{H} 5 \mathrm{~N} 1$ avian influenza viruses. J Virol 80:11115-11123.

Matrosovich MN, Gambaryan AS, Teneberg S, Piskarev VE, Yamnikova SS, Lvov DK, Robertson JS, Karlsson KA. 1997 Avian influenza A viruses differ from human viruses by recognition of sialyloligosaccharides and gangliosides and by a higher conservation of the HA receptor-binding site. Virology 233:224-234.

Matrosovich MN, Zhou N, Kawaoka Y, Webster R 1995 The surface glycoproteins of $\mathrm{H} 5$ influenza viruses isolated from humans, chickens, and wild aquatic birds have distinguishable properties. J Virol 73:1146-1155.

Munster VJ, Wallensten A, Baas C, Rimmelzwaan GF, Schu- 
tten M, Olsen B, Osterhaus AD, Fouchier RA 2005 Mallards and highly pathogenic avian influenza ancestral viruses, northern Europe. Emerg Infect Dis 11:1545-1551.

Obenauer JC, Denson J, Mehta PK, Su X, Mukatira S, Finkelstein DB, Xu X, Wang J, Ma J, Fan Y, Rakestraw KM, Webster RG, Hoffmann E, Krauss S, Zheng J, Zhang Z, Naeve CW 2006 Large-scale sequence analysis of avian influenza isolates. Science 311:576-580.

Qiu BF, Liu WJ, Peng DX, Hu SL, Tang YH, Liu XF 2009 A reverse transcription-PCR for subtyping of the neuraminidase of avian influenza viruses. J Virol Methods 155:193-198.

Seo SH, Hoffmann E, Webster RG 2002 Lethal H5N1 influenza viruses escape host anti-viral cytokine responses. Nat Med 8:950-954.

Shortridge KF, Zhou NN, Guan Y, Gao P, Ito T, Kawaoka Y, Kodihalli S, Krauss S, Markwell D, Murti KG, Norwood M, Senne D, Sims L, Takada A, Webster RG 1998 Characterization of avian $\mathrm{H} 5 \mathrm{~N} 1$ influenza viruses from poultry in Hong Kong. Virology 252:331-342.

Steel J, Lowen AC, Mubareka S, Palese P 2009 Transmission of influenza virus in a mammalian host is increased by PB2 amino acids $627 \mathrm{~K}$ or $627 \mathrm{E} / 701 \mathrm{~N}$. PLoS Pathog 5: e1000252.

Stieneke-Gröber A, Vey M, Angliker H, Shaw E, Thomas G, Roberts C, Klenk HD, Garten W 1992 Influenza virus hemagglutinin with multibasic cleavage site is activated by furin, a subtilisin-like endoprotease. EMBO J 11:2407-2414.

Tamura K, Dudley J, Nei M, Kumar S 2007 MEGA4: Molecular Evolutionary Genetics Analysis (MEGA) software version 4.0. Mol Biol Evol 24:1596-1599.

Tsukamoto K, Ashizawa H, Nakanishi K, Kaji N, Suzuki K, Okamatsu M, Yamaguchi S, Mase M 2008 Subtyping of avian influenza viruses $\mathrm{H} 1$ to H15 on the basis of hemagglutinin genes by PCR assay and molecular determination of pathogenic potential. J Clin Microbiol 46:30483055 .

Tumpey TM, Maines TR, Van Hoeven N, Glaser L, Solórzano A, Pappas C, Cox NJ, Swayne DE, Palese P, Katz JM, García-Sastre A 2007 A two-amino acid change in the hemagglutinin of the 1918 influenza virus abolishes transmission. Science 315:655-659.

Wang C, Takeuchi K, Pinto LH, Lamb RA 1993 Ion channel activity of influenza A virus M2 protein: characterization of the amantadine block. J Virol 67:5585-5594.

Yamada S, Suzuki Y, Suzuki T, Le MQ, Nidom CA, SakaiTagawa Y, Muramoto $\mathrm{Y}$, Ito $\mathrm{M}$, Kiso M, Horimoto $\mathrm{T}$, Shinya K, Sawada T, Kiso M, Usui T, Murata t, Lin Y, Hay A, Haire LF, Stevens DJ, Russell RJ, Gamblin SJ, Skehel JJ, Kawaoka Y 2006 Haemagglutinin mutations responsible for the binding of $\mathrm{H} 5 \mathrm{~N} 1$ influenza $\mathrm{A}$ viruses to human-type receptors. Nature 444:378-382.

(접수: 2013. 8. 26, 수정: 2013. 9. 17, 채택: 2013. 9. 17) 